\title{
OSCILLATING TRANSVERSE VOLTAGE IN A CHANNEL WITH QUANTUM POINT
}

\section{CONTACT VOLTAGE PROBES}

\author{
L.W. Molenkamp, H. van Houten, C.W.J. Beenakker, \\ R. Eppenga and C.T. Foxon* \\ Philips Research Laboratories \\ 5600 JA Eindhoven, The Netherlands \\ * Philips Research Laboratories \\ Redhill, Surrey, RH1 5HA, UK
}

\section{ABSTRACT}

We have observed a transverse voltage on passing a current through a narrow channel, electrostatically defined in a twodimensional electron gas, at zero magnetic field. The channel is fitted with two opposite quantum point contact voltage probes, and the voltage occurs when these probes are differently adjusted, so that the transmission probabilities through the probes have a different energy dependence. The transverse voltage occurs only in the nonlinear response regime, and is even in the applied current; the driving force of the effect is the current-heating of the electrons in the channel. We observe strong oscillations in the transverse voltage as the number of occupied subbands in one of the voltage probes is varied by means of electrostatic or magnetic depopulation. Model

calculations show that this novel effect is a manifestation of the oscillatory thermopower of a quantum point contact predicted by streda. The effect can thus be used to obtain information on electron heating.

\section{INTRODUCTION}

The quantized conductance of a short and narrow constriction (quantum point contact) in a two-dimensional electron gas (2DEG) was discovered only recently[1], but has already been utilized advantageously for the experimental study of a variety of fundamental transport phenomena[2]. Part of this research was aimed directly at the transport properties of the point contacts themselves. In the regime of linear response this work included, e.g., detailed studies of the ubiquity of the quantization at zero magnetic field[3] and experiments on the magnetic depopulation[4] of one-dimensional (1D) subbands at finite fields. In a later stage, the research was extended to the nonlinear regime, involving measurements and interpretation of the nonlinear I-V characteristics of a quantum point contact [5]. Subsequently, electronic instabilities occurring at high voltages across the point contact were reported[6]. 
Another class of experiments includes studies where two adjacent point contacts are used as voltage and current probes of ballistic transport phenomena. This approach was first used to study coherent electron focusing[7]. The electron focusing technique was subsequently used to study scattering processes[8] and to detect the injection of hot electrons through a voltage biased point contact[9]. At high magnetic fields, adjacent point contacts have been used for selective excitation and detection of the edge channels that are responsible for the transport in this regime, resulting in the observation of an anomalous quantization of the quantum Hall plateaus[10]. A geometry with two opposite point contacts was used to study the ballistic series resistance[11] and to detect electron beam collimation [12].

In this paper we demonstrate how one can use two opposite point contact voltage probes to detect electron heating in a current carrying channel. We will show that this detection technique constitutes a measurement of the thermopower of a quantum point contact, thereby enabling an estimate of the amount of electron heating in the channel. In addition, we observe strong oscillations that are related to the depopulation of $1 \mathrm{D}$ electric subbands in the quantum point contacts. Our main results have been briefly reported elsewhere[13].

\section{TRANSVERSE VOLTAGE QUADRATIC IN THE CURRENT}

Using electron beam lithography, we have fabricated splitgate microstructures on a GaAs/( $A l, G a)$ As heterojunction wafer with a 2DEG mobility of about $100 \mathrm{~m}^{2} \mathrm{~V}^{-1} \mathrm{~s}^{-1}$ and a carrier density $\mathrm{n}_{\mathrm{s}}=3.5 \times 10^{15} \mathrm{~m}^{-2}$. The structures consist [12] of a narrow channel of $18 \mu \mathrm{m}$ length and a width $\mathrm{w}_{0}$ of $4 \mu \mathrm{m}$. On both sides of the channel two point contacts are defined, with $3 \mu \mathrm{m}$ separation. A schematic layout of the gates and ohmic contacts is given in Fig. 1. Unless stated otherwise, the samples are kept at a temperature of $1.65 \mathrm{~K}$ and no magnetic field is applied.

We have performed a number of experiments to study the electron heating in the channel. In a first experiment, a dc

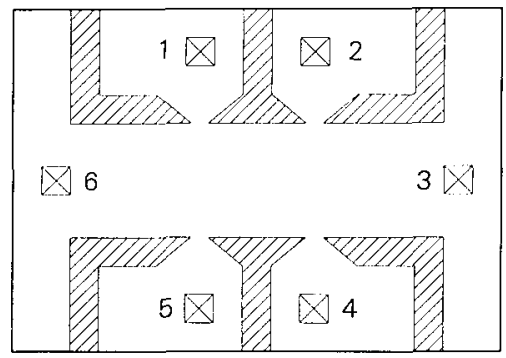

Fig. 1. Schematical layout of the samples used in these experiments. The hatched areas indicate the top gates, the crosses depict the ohmic contacts. In the actual devices, the channel has a length of $18 \mu \mathrm{m}$ and a width $\mathrm{w}_{0}$ of $4 \mu \mathrm{m}$. The separation between two adjacent point contacts is $3 \mu \mathrm{m}$. 


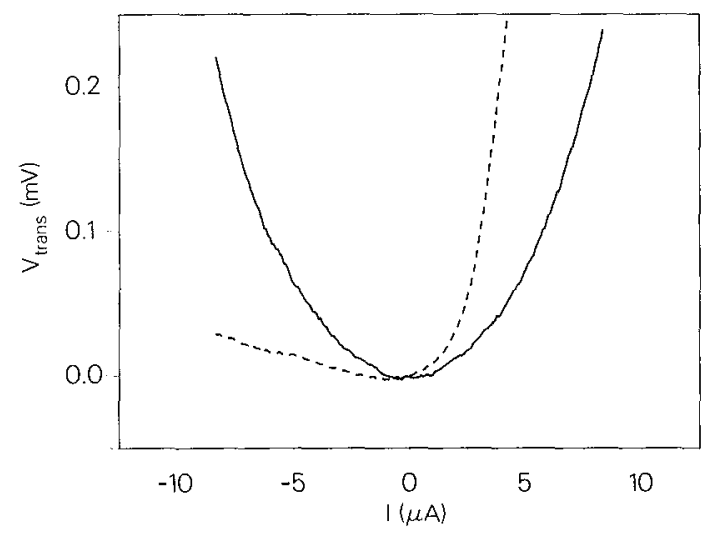

Fig. 2. The dependence of $V_{\text {trans }}$ on the current in the channel. In this plot, $\mathrm{V}_{\text {gate }}=-2.97 \mathrm{~V}$, corresponding to $R_{p c} \approx 12.9 \mathrm{k} \Omega$, and $V_{\text {gate }} 5=$ $-0.7 \mathrm{~V}\left(\mathrm{R}_{\mathrm{pc}}=0.5 \mathrm{k} \Omega\right)$. The lattice temperature $\mathrm{T}_{0}=1.65 \mathrm{~K}$. The drawn (dashed) curve was obtained using ohmic contacts 6 and $3(2)$ as current source and drain, respectively. (In these experiments we have not averaged over both current directions.)

current I is passed through the channel, using 2DEG contacts 6 and 3 as source and drain, respectively. We now measure the voltage on contact 5 relative to that on contact 1 , i.e. a transverse voltage $v_{\text {trans }} \equiv v_{5}-v_{1}$, where we have adjusted the gates such that the resistance of point contact 1 (leading to voltage probe 1) is much higher than that of point contact 5 . As discussed below, electron heating leads to a positive value of $V_{\text {trans }}$ due to accumulation of electrons in the $2 \mathrm{DEG}$ region behind the most strongly pinched-off point contact (1). The curve in Fig. 2 is a plot of $V_{t r a n s}$ as a function of the current $\mathrm{I}$, for $\mathrm{V}_{\text {gate }}=-2.97 \mathrm{~V}, \mathrm{~V}_{\text {gate }}=-0.7 \mathrm{~V}$ and $\mathrm{I}$ ranging between -10 and $+10 \mu \mathrm{A}$. The observation of a nonzero $V_{t r a n s}$ in the absence of a magnetic field is by symmetry not allowed in the linear response regime. The observed $V_{t r a n s}$ exhibits, to a very good approximation, a quadratic dependence upon $I$. It is one of the very few examples of even-order nonlinear behavior reported so far in semiconductor microstructures. Previously, a second order nonlinearity was observed as second harmonic generation in the quantum diffusive transport regime at mK temperatures[1416]. This effect saturated at very low current levels - four orders of magnitude lower than those relevant for our structures. We have observed the quadratic current dependence in this configuration for currents up to $20 \mu \mathrm{A}$ and temperatures up to $30 \mathrm{~K}$.

Since our signal is relatively large and robust, it is evident that the observed behavior is not related to quantum coherence in the channel. We attribute the effect to current heating of the electrons in the channel. In a homogeneous, isotropic material, Joule heating can only lead to odd-order nonlinearities in the longitudinal voltage response $[16,17]$. The reason is one of symmetry: a longitudinal voltage has to change sign on reversing the current. The even-order nonlinearity 


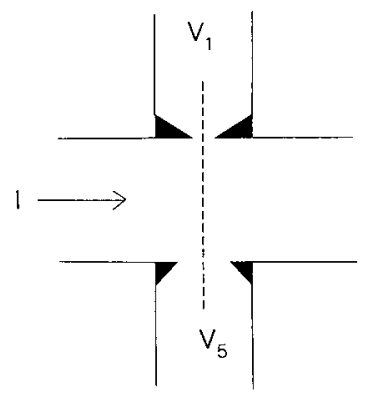

Fig. 3. An arrangement for transverse voltage measurements in a channel. The point contact voltage probes are indicated in black. The dashed line is a line of mirror symmetry; its presence demands that the transverse voltage should be even in the current.

observed here is due to the effect of the voltage probes themselves on the measured voltage difference. The measurement geometry is shown schematically in Fig. 3. As is evident from this figure, symmetry now requires that any transverse voltage $V_{5}-V_{1}$ should be even in the current.

Assuming Joule heating as the driving force of our effect, the simplest classical mechanism leading to a transverse voltage emerges when one realizes that in a point contact the bottom of the conduction band is raised with respect to the $2 \mathrm{DEG}$ regions leading to the point contact[2]. Hot electrons in the channel will easily overcome this barrier and enter the cold regions behind the point contacts. Since we use the point contacts as voltage probes, this flux must be compensated by a flux of cold electrons back into the channel. These cold electrons can only cross the energy barrier of the point contact provided the cold 2DEG region is sufficiently charged up. We thus expect a voltage to develop across the point contacts which depends on the energy barrier height, and thus on the resistance of the point contacts. To the extent that the heated electrons in the channel can be described by a Fermi-Dirac distribution, our transverse voltage becomes simply the difference in thermovoltage of two differently adjusted point contacts. While the classical mechanism described above is qualitatively correct, it cannot explain the oscillations in the transverse voltage discussed in the following section. A quantum mechanical treatment is necessary to account for all our observations, and will be given below.

In view of our claim that Joule heating causes the transverse voltage, it is important to estimate the actual temperature of the electrons in the channel. We assume that the lattice temperature $\mathrm{T}_{0}$ is unaffected and uniform over the sample. A rough indication of the electron temperature $T$ in the channel can be obtained from the heat-balance equation

$$
c_{v}\left(T-T_{0}\right)=\left(I / W_{0}\right)^{2} \rho \tau_{\epsilon}
$$

where $c_{V}=\left(\pi^{2} / 3\right)\left(k_{B} T / E_{F}\right) n_{S} k_{B}$ is the heat capacity per unit area of the $2 \mathrm{DEG}, \rho$ is the resistivity in the channel, and $\tau_{\epsilon}$ an 
energy relaxation time associated with energy transfer from the electron gas to the lattice. For $I=5 \mu \mathrm{A}$ and an estimated[18] $\tau_{\epsilon} \approx 10^{-10}$ s this yields $\mathrm{T}-\mathrm{T}_{0} \approx 1 \mathrm{~K}$. A quantitative

calculation of the electron temperature should account for the temperature dependence of $C_{v}$ and $\tau_{\epsilon}$, and should also include the heat conduction in the 2DEG for the actual device geometry. An additional contribution of comparable magnitude to $\mathrm{T}-\mathrm{T}_{0}$ results from the contact resistance $R_{p c}$ of about $200 \Omega$. This result can be readily obtained from the expression for the Sharvin resistance [2], $R_{p c}=\left(h / 2 e^{2}\right)\left(\pi / k_{F} w_{0}\right)$, which leads to a voltage drop $I_{\mathrm{pc}} / 2$ at the entrance of the channel. (Note that this mechanism allows for the observation of a transverse voltage in a channel that is much shorter than the mean free path of the electrons). The magnitude of $V_{t r a n s}$ in the present geometry appears to saturate for currents larger than $20 \mu \mathrm{A}$. This is probably due to the temperature dependence of $c_{v}$ and $\tau_{\epsilon}$ (cf. Eqn. 1), in addition to nonnegligible lattice heating.

A larger $V_{\text {trans }}$ for a given current can be realized by injecting the current over a barrier. This leads to a large voltage drop close to the detecting voltage probes; in other words, the contact resistance contribution to the electron heating process becomes dominant. The dashed curve in Fig. 2 was obtained for the same gate voltages as used for the full curve, but now using contacts 6 and 2 as current source and drain, respectively. The enhancement of $V_{t r a n s}$ when the electrons are accelerated by the voltage drop over point contact 6 (which has a resistance of approximately $10 \mathrm{k} \Omega$ ) is quite dramatic. of course, in this case the hot electron distribution may differ appreciably from a heated Fermi-Dirac distribution, in which case $V_{t r a n s}$ is not simply related to the thermopower of the voltage probes. Note also that $V_{t r a n s}$ is not even in $I$ in this particular measurement geometry. The transverse voltage is only enhanced for electrons that first are accelerated over the barrier and then detected by the point contact voltage probes. In the reverse current direction, the electrons are heated after passing the voltage probes and $V_{\text {trans }}$ does not increase.

\section{QUANTUM OSCILLATIONS IN $V_{\text {trans }}$}

To further elucidate the origin of $V_{\text {trans }}$ we have performed a series of experiments using the same geometry as described above (the current I is passed through ohmic contacts 6 and 3 , and the voltage measured is $v_{\text {trans }}=V_{5}-V_{1}$ ), but now with a fixed current of $\mathrm{I}$ of $5 \mu \mathrm{A}$. We scan the voltage of the gates leading to contact 1 , while keeping the voltage on the other gates fixed at $-2.0 \mathrm{~V}$. To eliminate any spurious longitudinal resistance contributions (which occur in some samples due to a small misalignment of the voltage probes) the signal is averaged over both current directions. The full curve in Fig. $4 \mathrm{a}$ is the result of such an experiment. Dramatic oscillations in $v_{t r a n s}$ are observed, that are not expected from the above classical description. For comparison, we have plotted the gate voltage dependence of the (two-terminal) resistance of the scanned point contact (1) in the same figure (dashed line). The oscillations in $V_{t r a n s}$ peak whenever the resistance of the point contact changes from one plateau to another. From the expression[2] for the quantized resistance of a point contact, $R_{p c}=h / 2 e^{2} N\left(E_{F}\right)$, we see that this occurs whenever the number $N\left(E_{f}\right)$ of $1 D$ electric subbands in the quantum point contact that are available for 


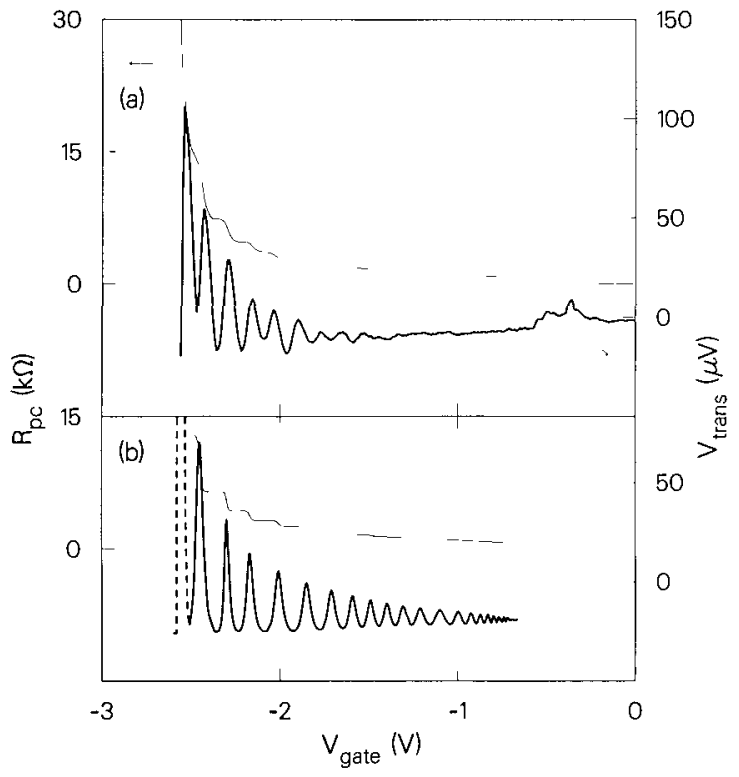

Fig. 4. (a) Experimental traces of $V_{\text {trans }}$ (thick curve) and $R_{p c}$ (thin curve) as a function of $V_{g a t e}$ at $a$ lattice temperature $\mathrm{T}_{0}=1.65 \mathrm{~K}$ for $\mathrm{I}=5 \mu \mathrm{A}$ and $V_{\text {gate }}=-2.0 \mathrm{~V}$. These data were obtained from a different sample than in Fig. 2. (b) Calculation of the transverse voltage (thick curve) using Eqns. 1-3 with electron temperature $\mathrm{T}=4 \mathrm{~K}, \mathrm{~T}_{0}=1.65 \mathrm{~K}$, and $\mathrm{E}_{\mathrm{F}}=13 \mathrm{meV}$. The thin curve gives the dependence of $R_{p c}$ on $V_{g a t e}$, calculated for a temperature $\mathrm{T}_{0}$ using experimental values of $\mathrm{W}$ and $\mathrm{E}_{0}$.

electrons at the Fermi energy $E_{F}$, changes by one. Below we will present a quantitative model, which will demonstrate explicitly the close relation between $V_{t r a n s}$ and the thermopower of $a$ quantum point contact.

To model our experiment we straightforwardly extend Streda's calculation[19] of the thermopower of a quantum point contact to finite temperature and voltage differences. (The method to calculate the thermopower from the transmission probabilities was developed by Sivan and Imry[20], along the lines of Landauer's formula for the conductance[21].) As the voltage probes draw no net current, the influx of hot electrons $I_{\text {hot } \rightarrow \text { cold }}$ from the channel into the voltage probe should exactly cancel the flux of cold electrons in the reverse direction, $I_{\text {cold hot }}$. We thus have $I_{\text {hot } \rightarrow \text { cold }}-I_{\text {cold } \rightarrow \text { hot }}=0$, or

$$
2-\int_{h}^{e} t(E)\left[f_{h o t}(E)-f_{c 01 d}(E)\right] d E=0
$$

where $t(E)$ is the transmission probability summed over the 1D subbands that propagate through the point contact at energy $\mathrm{E}$, and $f_{\text {hot }}$ and $f_{\text {cold }}$ are the distribution functions of the electrons in the channel and in the cold 2DEG region. For the 
present, we assume that these functions are well represented by Fermi-Dirac distributions at chemical potentials $E_{F}$ and $E_{F}+\Delta \mu$, and at temperatures $\mathrm{T}$ and $\mathrm{T}_{0}$, respectively. Note that Eqn. 2 holds for any hot electron distribution function which depends on energy only, so that it can also be used, e.g., to describe the effects of injection over a barrier, as discussed in the previous section. The quantum point contact is modeled by a square well lateral confinement potential of width $\mathrm{W}$ and well bottom at energy $\mathrm{E}_{0}$ (measured with respect to the conduction band bottom in the channel). Assuming a transmission of unity for each of the $N(E)$ subbands in the point contact, we have

$$
t(E)=N(E)=\operatorname{Int}\left[\left(2 m / \hbar^{2}\right)^{1 / 2}\left(E-E_{0}\right)^{1 / 2} W / \pi\right] \theta\left(E-E_{0}\right),
$$

where Int denotes truncation to an integer, and $\theta(x)$ is the unit step function. From Eqns. 2 and 3 we can obtain $\Delta \mu$ numerically. Since $V_{\text {trans }}$ is the difference of the voltage measured by two differently adjusted point contacts, the above calculation should be repeated for the reference point contact, to obtain $\Delta \mu^{\text {ref }}$, which has a constant value. The transverse voltage is then found from $V_{\text {trans }}=\left(\Delta \mu-\Delta \mu^{r e f}\right) / e$. For comparison with the experiment, we have treated $\Delta \mu^{r e f}$ as an adjustable baseline.

The result of our calculation, for $\mathrm{T}_{0}=1.65 \mathrm{~K}$ and assuming $T=4 \mathrm{~K}$ (consistent with the estimate from the heat balance equation discussed above for a current $I=5 \mu \mathrm{A})$, is given in Fig. 4b. Experimentally determined values for $W$ and $E_{0}$ were used. The good agreement of the calculated curve with the experimental data of Fig. 4 a indicates that our theoretical understanding of the effect is basically correct. No detailed quantitative agreement is obtained, and was not to be expected. For example, the peaks in the experiment are broader than the theoretical ones. Similarly, the experimental point contact resistance $R_{p c}$ shows less pronounced steps than the calculation. Since both $R_{p c}$ and $V_{t r a n s}$ depend on the detailed behavior of $t(E)$ near $E_{F}$, both discrepancies may be ascribed, at least partly, to uncertainties in the transmission probability. The experiments show additional structure around threshold ( $\mathrm{V}_{\text {gate }}=-0.5 \mathrm{~V}$ ) where the point contact (and the channel) is just defined. is explained by the associated abrupt change[2] in $t\left(E_{F}\right)$. The voltage peak near $V_{\text {gate }} \approx-2.6 \mathrm{~V}$ (just beyond the $\mathrm{R}=\mathrm{h} / 2 \mathrm{e}^{2}$ resistance plateau), turns out much weaker in the experiment than in our calculations (dashed part). The size of this peak is very sensitive to the (unknown) details of the dependence of $t(E)$ on $V_{g a t e}$ in the pinch-off regime, and we have not attempted to achieve a better agreement.

The connection of the above calculation with streda's result on the thermopower of a quantum point contact can be made explicit on considering the limiting behavior at low lattice temperatures and small electron heating $\left(k_{B} T_{0}\right.$ and $k_{B} T$ both much smaller than the subband separation at the Fermi energy). In this limit, Eqns. 2 and 3 yield the result[19] that the peak in $\Delta \mu$ when the $(\mathrm{N}+1)-$ th subband is depleted has amplitude $\Delta \mu \approx(\ln 2) \mathrm{k}_{\mathrm{B}}\left(\mathrm{T}-\mathrm{T}_{0}\right) / \mathrm{N}$. The transverse voltage experiment employing current heating is a very convenient way to measure the thermopower in the quantum ballistic transport regime, where only temperature differences on the scale of a mean free path are important. Application of an external temperature difference to a semiconductor sample containing a quantum point contact would only result in a very small temperature drop over 
the point contact, and the signal would be obscured by spurious contributions from the thermopower of the bulk 2DEG, enhanced by phonon drag contributions[22]. Since the lattice temperature $\mathrm{T}_{0}$ is kept uniform in the present experiments, phonon drag does not play a role. Current heating was very recently also used for the study of universal thermopower fluctuations in the phase coherent diffusive transport regime[23].

\section{QUANTUM OSCILLATIONS AT MODERATE MAGNETIC FIELD}

Using the same measurement geometry as described above, we have also performed measurements of the quantum oscillations in moderate magnetic fields. The results of such experiments, for magnetic fields of $0,0.75,1.5$ and $3.0 \mathrm{~T}$ are shown in Fig. 5 . In a moderate perpendicular magnetic field, the number of 1D subbands in a point contact is determined both by the electrostatic potential of the gates and by the magnetic field[2]. The intersubband energy spacing of these magnetoelectric subbands increases with increasing magnetic field. Consequently, the number of occupied $1 \mathrm{D}$ subbands $N\left(E_{F}\right)$ for a given Fermi energy $E_{F}$

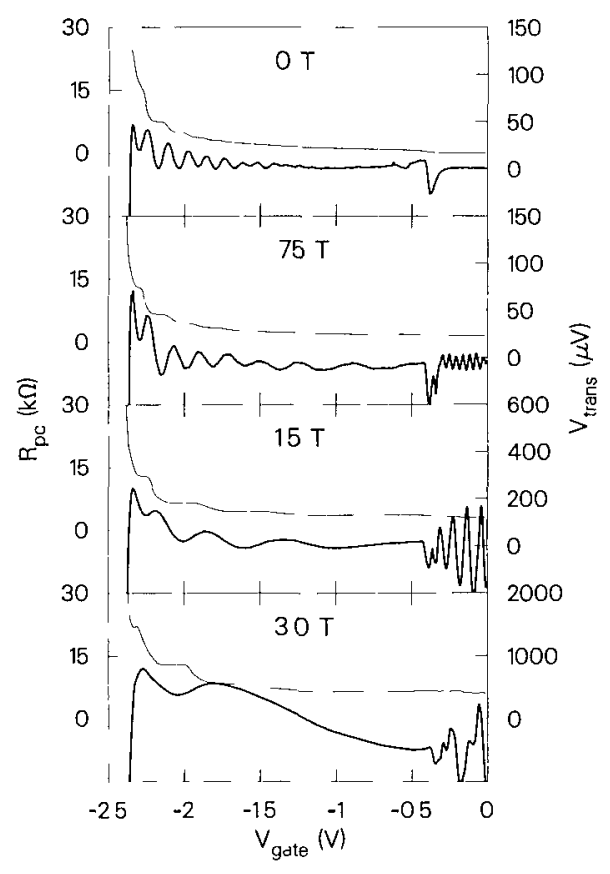

Fig. 5. The effects of magnetic depopulation on $V_{t r a n s}$ : Again, the thick curves give $V_{t r a n s}$ and the thin curves $R_{p c}$. The magnetic fields at which the data were taken are indicated in the figures. other experimental conditions: $\mathrm{T}_{0}=1.65 \mathrm{~K}$, $\mathrm{I}=5 \mu \mathrm{A}$ and $\mathrm{V}_{\mathrm{gate}} 5=-1.0 \mathrm{~V}$. The rapid oscillations at small gate voltages are due to depopulation of the Landau levels in the 2DEG area underneath the gate. The data were taken from the same sample as in Fig. 4; however, due to thermal cycling the zero-field dependence of $R_{p c}$ on $V_{g a t e}$ has changed. 
decreases with increasing magnetic field. This magnetic depopulation effect can be very directly observed by measuring the conductance of a quantum point contact as a function of gate voltage, for various magnetic fields. The magnetic depopulation manifests itself by a reduction of the number of plateaus in a given gate voltage interval[4].

Magnetic depopulation of 1D subbands in the quantum point contact affects the oscillations in $V_{t r a n s}$ as a function of $V_{g a t e}$ in a very similar manner: in a magnetic field, $V_{\text {trans }}$ still exhibits peaks at gate voltages where the number of magnetoelectric subbands changes by one, but the oscillations are more widely spaced in gate voltage. The increase in magnitude of $V_{\text {trans }}$ at the magnetic field values used in Fig. 5 is caused by the enhancement of the longitudinal resistivity $\rho_{x x}$ due to Shubnikov-de Haas oscillations and, hence, of the power dissipation in the channel.

In the traces taken at finite magnetic fields, additional rapid oscillations are seen in Fig. 5 at small gate voltages where one point contact and one of the channel boundaries are not yet fully defined. For $V_{g a t e}^{d e f}<V_{g a t e}<0$, where $V_{g a t e}^{d e f}$ is the depletion threshold under the gate, the partially depleted 2DEG area underneath the gates acts as a weak potential barrier for the electrons moving from the channel region to the $2 \mathrm{DEG}$ region surrounding the ohmic contacts. Thus, a transverse voltage will develop on passing a current through the channel, analogous to the situation discussed in the previous paragraphs where the point contact acts as potential barrier. The rapid oscillations as a function of gate voltage can thus be explained by the stepwise decrease in the number of Landau levels in the barrier region. (Because of the absence of lateral confinement, the relevant quantum states in the wide regions under the gate are bulk Landau levels, rather than 1D magnetoelectric subbands[2].) Note that the trace taken at $3.0 \mathrm{~T}$ shows a doublet structure, presumably due to spin splitting.

The rapid oscillations are linear in $\mathrm{V}_{9}$ ate, which implies that the density of the 2DEG underneath the gate is also linear in $V_{\text {gate }}$ (as expected when the capacitance between gate and 2DEG does not depend on $\left.V_{g a t e}\right)$. We can estimate the carrier density under the gate at zero gate voltage, $n_{s}^{g a t e}$, by measuring the period $\Delta v_{g}$ ate of the oscillations in this gate voltage region. We use

$$
\nu=\frac{h n_{s}^{\text {gate }}}{2 \mathrm{eB}}=\frac{V_{g a t e}^{\text {def }}}{\Delta V_{\text {gate }}}
$$

where $\nu$ is the filling factor (the factor of two accounts for the two spin states). From Fig. 5 we find that for all three fields $n_{s}^{g a t e} \approx 3.3 \times 10^{15} \mathrm{~m}^{-2}$, which is only slightly smaller than the density of the bulk 2 DEG $\left(n_{s} \approx 3.5 \times 10^{15} \mathrm{~m}^{-2}\right)$, which was determined from Shubnikov-de Haas measurements on the same sample. The electron concentration in the $2 \mathrm{DEG}$ is thus hardly affected by the presence of a grounded top gate. 
We have demonstrated a novel type of nonlinearity in a semiconductor nanostructure, caused by electron heating. In contrast to thermal effects in bulk material, where only oddorder nonlinearities in the longitudinal voltage are expected, we find a transverse voltage that is even in the current. The essential requirement to observe such a voltage is a difference in transmission probability for hot and cold electrons in at least one of the voltage probes. The origin of this difference can be classical (a potential barrier in the probes), or quantum mechanical (discrete 1D subbands in the probes). The presence of point contact voltage probes in our channel has enabled us to observe the quantum oscillations in the thermopower of the point contacts[19]. Other applications in physics might include the determination of hot electron distributions in spatial directions that are not accessible to conventional hot electron spectroscopy[24]. Finally, one can anticipate that this method may be used as a sensitive probe for the study of magnetothermal effects, such as magnetophonon resonances. Since the effect is not fundamentally limited to very low temperatures, its application in devices, e.g., high frequency mixers and parametric amplifiers is well worth pursuing.

\section{Acknowledgment}

We acknowledge C.E. Timmering for sample preparation, M.A.A. Mabesoone for technical assistance during the experiments, and A.A.M. Staring, J.G. Williamson, and M.F.H. Schuurmans for valuable discussions.

\section{REFERENCES}

1. B.J. van Wees, H. van Houten, C.W.J. Beenakker, J.G. Williamson, L.P. Kouwenhoven, D. van der Marel and C.T. Foxon, Phys. Rev. Lett., 60:848 (1988); D.A. Wharam, T.J. Thornton, R. Newbury, M. Pepper, H. Ahmed, J.E.F. Frost, D.G. Hasko, D.C. Peacock, D.A. Ritchie and G.A.C. Jones, J. Phys. C, 21:L209 (1988)

2. H. van Houten, C.W.J. Beenakker and B.J. van Wees, in: "Semiconductors and Semimetals", M. Reed, ed., Academic Press, New York, to be published.

3. G. Timp, in: "Semiconductors and Semimetals", M. Reed, ed., Academic Press, New York, to be published.

4. B.J. van Wees, L.P. Kouwenhoven, H. van Houten, C.W.J. Beenakker, J.E. Mooij, C.T. Foxon and J.J. Harris, Phys. Rev. B, 38:3625 (1988)

5. L.P. Kouwenhoven, B.J. van Wees, C.J.P.M. Harmans, J.G. Williamson, H. van Houten, C.W.J. Beenakker, C.T. Foxon and J.J. Harris, Phys. Rev. B, 39:8040 (1989)

6. R.J. Brown, M.J. Kelly, M. Pepper, H. Ahmed, D.G. Hasko, D.C. Peacock, J.E.F. Frost, D.A. Ritchie and G.A.C. Jones, J. Phys. Condens. Matter, 1:6285 (1989)

7. H. van Houten, B.J. van Wees, J.E. Mooij, C.W.J. Beenakker, J.G. Williamson and C.T. Foxon, Europhys. Lett., 5:721

(1988); H. van Houten, C.W.J. Beenakker, J.G. Williamson, M.E.I. Broekaart, P.H.M. van Loosdrecht, B.J. van Wees, J.E. Mooij, C.T. Foxon and J.J. Harris, Phys. Rev. B, $39: 8556(1989)$

8. J. Spector, H.L. Stormer, K.W. Baldwin, L.N. Pfeiffer and 
K.W. West, surf. Sci., 228:283 (1990)

9. J.G. Williamson, H. van Houten, C.W.J. Beenakker, M.E.I. Broekaart, L.I.A. Spendeler, B.J. van Wees and C.T. Foxon, Phys. Rev. B, 41:1207 (1990)

10. B.J. van Wees, E.M.M. Willems, C.J.P.M. Harmans, C.W.J. Beenakker, H. van Houten, J.G. Williamson, C.T. Foxon and J.J. Harris, Phys. Rev. Lett., 62:1181 (1989)

11. D.A. Wharam, M. Pepper, H. Ahmed, J.E.F. Frost, D.G. Hasko, D.C. Peacock, D.A. Ritchie and G.A.C. Jones, J. Phys. C, $21: L 887$ (1988)

12. L.W. Molenkamp, A.A.M. Staring, C.W.J. Beenakker, R. Eppenga, C.E. Timmering, J.G. Williamson, C.J.P.M. Harmans and C.T. Foxon, Phys. Rev. B, 41:1274 (1990)

13. L.W. Molenkamp, H. van Houten, C.W.J. Beenakker, R. Eppenga and C.T. Foxon, Phys. Rev. Lett., 65:1052 (1990)

14. R.A. Webb, S. Washburn and C.P. Umbach, Phys. Rev. B, $37: 8455$ (1988)

15. S.B. Kaplan, Surf. Sci., 196:93 (1988)

16. P.G.N. de Vegvar, G. Timp, P.M. Mankiewich, J.E. Cunningham, R. Behringer and R.E. Howard, Phys. Rev. B, $38: 4326$ (1988)

17. R. Landauer, in: "Nonlinearity in Condensed Matter", A.R. Bishop, D.K. Campbell, P. Kumar and S.E. Trullinger, eds., Springer, Berlin (1987)

18. D.R. Leadley, R.J. Nicholas, J.J. Harris and C.T. Foxon, Solid state Electronics, 32:1473 (1989)

19. P. Streda, J. Phys. Condens. Matter, 1:1025 (1989)

20. U. Sivan and Y. Imry, Phys. Rev. B, 33:551 (1986)

21. R. Landauer, IBM J. Res. Dev., 1:223 (1957)

22. R. Fletcher, M. D'Iorio, A.S. Sachrajda, R. Stoner, C.T. Foxon and J.J. Harris, Phys. Rev. B, 37:3137 (1988); C. Ruf, H. Oblow, B. Junge, E. Gmelin, K. Ploog and G. Weimann, Phys. Rev. B, 37:6377 (1988)

23. B.L. Gallagher, T. Galloway, P. Beton, J.P. Oxley, S.P. Beaumont, S. Thoms and C.D.W. Wilkinson, Phys. Rev. Lett., $64: 2058$ (1990)

24. See, e.g. J.R. Hayes, A.F.J. Levi and W. Wiegman, Phys. Rev. Lett., $54: 1570$ (1985); A. Palevski, M. Heiblum, C.P. Umbach, C.M. Knoedler, A.N. Broers and R.H. Koch, Phys. Rev. Lett., 62:1776 (1989) 